\title{
Standardizing thoracic organ procurement for transplantation
}

\author{
Michael K. Pasque, MD
}

In direct contrast to the transplant procurement of abdominal solid organs, in which transplant surgeons routinely accept organs that have been harvested by other surgical groups, there has been a general lack of enthusiasm for sharing organ procurement responsibilities among thoracic transplant surgeons. This has resulted in reduced donor organ usage, increased expense in an already expensive venture, and inefficiency in human resource allocation in the treatment of end-stage cardiac and pulmonary dysfunction. The elimination of variation in thoracic organ procurement technique is the mandatory first step in a much-needed dialogue aimed at improving thoracic organ procurement efficiency. Toward this end, a standardized procedure for adult heart and lung donor organ procurement is proposed.

\section{COMBINED HEART AND LUNG PROCUREMENT Initial On-Site Donor Evaluation}

- Upon arrival at the donor medical facility, donor/recipient blood typing and compatibility are confirmed with a review of all pertinent donor history.

- The donor hemodynamics, inotropic/pressor infusion rates, electrocardiogram, echocardiogram, and cardiac catheterization (if obtained) are reviewed. The latest arterial blood gas data and chest $\mathrm{x}$-ray are reviewed and bronchoscopy is performed. Any unanticipated cardiac or pulmonary abnormalities are immediately reported to the respective recipient implant team.

- Access for a standard median sternotomy is gained through a Y-shaped midline skin incision that spares the neck and upper chest.

This skin incision allows more options for open casket funerals and is requested by funeral homes, appreciated by families, and mandated by some organ procurement organizations.

- Both pleural spaces are entered without cautery to avoid inadvertent burn injury to the lungs. The lungs are palpated and visually examined. The results of this examination, as well as the latest results of the arterial blood gas, chest radiograph, and bronchoscopic examinations, are

\footnotetext{
From the Division of Cardiothoracic Surgery, Washington University School of Medicine, Barnes-Jewish Hospital, St Louis, Mo.

Received for publication Aug 4, 2009; accepted for publication Sept 9, 2009.

Address for reprints: Michael K. Pasque, MD, Division of Cardiothoracic Surgery, Washington University School of Medicine; Barnes-Jewish Hospital, One Barnes-Jewish Hospital Plaza, 3103 Queeny Tower, St Louis, MO 63110 (E-mail: PASQUEM@WUSTL.EDU).

J Thorac Cardiovasc Surg 2010;139:13-7

0022-5223/\$36.00

Copyright $₫ 2010$ by The American Association for Thoracic Surgery

doi:10.1016/j.jtcvs.2009.09.015
}

immediately called to the lung recipient implant team. The anticipated crossclamp time is also updated.

- Recruitment of all atelectatic lung segments is carried out in situ by the gentle massage of both lungs as the anesthesiologist inflates them to a sustained $30 \mathrm{~cm} \mathrm{H}_{2} \mathrm{O}$ pressure.

The practice of sequentially eviscerating the lungs from the pleural cavity to perform recruitment of the atelectatic areas of the lungs is strongly discouraged. In situ recruitment is more than adequate to clear atelectasis and lessens the possibility of severe lung hyperinflation, injury to hilar structures, and the hemodynamic compromise that is always associated with unilateral lung evisceration. Although the routine donor may tolerate short periods of transient hemodynamic compromise, the downward spiral that can be initiated by such maneuvers in the borderline donor can place all organs-and thus their potential recipients-at immediate and unnecessary risk.

- A pericardial well is created while direct access to both pleural spaces is maintained.

- The heart and great vessels are examined for congenital anatomic abnormalities, traumatic injury, unanticipated atherosclerotic coronary artery disease, palpable thrills, and biventricular contractile dysfunction. The results of this examination are called to the heart recipient implant team along with an update on the latest donor hemodynamics, inotropic/pressor infusion rates, and the anticipated crossclamp time.

\section{THORACIC ORGAN DISSECTION}

- The main and right pulmonary arteries are carefully separated from the ascending aorta.

- The attachments of the superior vena cava to the right pulmonary artery are divided. The superior vena cava is dissected free of all circumferential mediastinal attachments up to its bifurcation into the innominate veins.

- A heavy silk ligature is passed around the superior vena cava cephalad to the azygos vein.

- At the discretion of the procurement surgeon, the azygos vein can be ligated at this time. It does not need to be divided.

The practice of routine division of the azygos vein by inexperienced operators has resulted in a significant incidence of unnecessary hemorrhage, as well as damage to the adjacent upper lobe branch of the right pulmonary artery. In most cases, an adequate length of superior vena cava can easily be mobilized without actually dividing this fragile structure. 
- Careful sharp dissection of the Sondergaard (Waterston) interatrial groove (to allow easier subsequent differentiation of cardiac and pulmonary left atrial cuffs) is then performed.

- A horizontal mattress 4-0 polypropylene suture (Prolene; Ethicon, Inc, Somerville, NJ) is then placed in the anterior midportion of the ascending aorta to later secure the cardioplegia cannula.

- A similar "pulmoplegia" cannulation suture is placed in the distal main pulmonary artery.

The pulmonary artery cannula site should be a minimum of $1.5 \mathrm{~cm}$ from the pulmonary valve to guarantee a generous cuff of main pulmonary artery for the subsequent cardiac implantation procedure. Placement near or even at the bifurcation of the main pulmonary artery still leaves more than enough right and left pulmonary artery length for bilateral lung transplantation. Inasmuch as the pulmoplegia cannula is being placed in the distal main pulmonary artery, the tip of the cannula is pointed backward toward the pulmonary valve. This prevents the preferential perfusion of one lung over the other that may occur if the tip is directed posteriorly toward the bifurcation of the main pulmonary artery.

- Incising the posterior pericardium between the superior vena cava and ascending aorta (just cephalad to the right pulmonary artery) allows the gentle dissection of the trachea above the carina. The anterior, lateral, and medial surfaces of the trachea can be cleared to allow more rapid tracheal access during the actual lung block excision.

It is not necessary to dissect the fascial attachments between the trachea and esophagus at this time. Overzealous manipulation can dislodge the endotracheal tube. In addition, attempts to bluntly divide the fascial connections between the trachea and esophagus at this stage of the procedure may result in esophageal perforation and mediastinal soiling.

\section{THORACIC ORGAN PRESERVATION}

- When the dissection of all donor organs has been completed to the satisfaction of the procurement surgeons, the organ preservation and excision process is initiated by the administration of heparin (30,000 units) through central venous access.

- A minimum of 3 minutes after heparin administration, the cardioplegia cannula and pulmoplegia cannulas are placed and connected to their respective infusion tubing.

The cardiac preservation infusion is given through a standard cardioplegia cannula. The lung perfusate is given via a high-flow 6.5-mm metal-tip cannula.

- A final in situ recruitment of atelectatic lung tissue can be carried out at this point if significant pulmonary atelectasis has again accumulated.
- The thoracic organ preservation procedure is initiated by the bolus administration of prostaglandin $\mathrm{E}_{1}$ (Alprostadil) into the main pulmonary artery immediately adjacent to the pulmoplegia cannula.

- The superior vena cava is ligated with the previously placed supra-azygos silk ligature.

- The left atrium is vented by transecting the tip of the left atrial appendage.

This transection should be in the midportion of the left atrial appendage to assure that the vent hole is adequate to prevent distention of the left ventricle by the pulmoplegia solution returning from the pulmonary veins. Care is taken to leave a large safe margin of left atrial appendage cuff such that its later closure by the implanting cardiac surgeon will not compromise the nearby circumflex coronary artery. Because of the proximity of the left atrial appendage to vital structures, its transection leaves little room for technical error. Optimal visualization for this critical part of the procurement procedure can be assured by performing this maneuver before the pericardium is flooded by the torrent of blood that is released by the venting of the inferior vena cava. This ordering of venting maneuvers renders unnecessary the practice of placing a vascular occlusion clamp on the left atrial appendage to allow its transection before initiating organ preservation. The placement of a rigid vascular clamp on the fragile left atrial appendage is to be strongly discouraged because the vigorous contractile movement of the heart in the confines of the pericardium applies significant shearing force to the fragile appendage tissue. This shearing force can result in a tear down into the base of the appendage, thereby compromising its subsequent closure and thus the integrity of the circumflex coronary artery.

- Alternatively, the left atrium can be vented by making the initial portion of the left atrial incision between the atrioventricular groove and the left inferior pulmonary vein. This incision will later be extended during the division of the left atrium into cardiac and pulmonary cuffs.

Experience with the use of this left atrial incision for venting has demonstrated that it often fails to adequately vent the left atrium (resulting in left ventricular distention) unless its initial length is at least $3 \mathrm{~cm}$. The obvious advantage of this technique is the elimination of the need for suture closure of the left atrial appendage vent site.

- Alternatively, the left atrium can be vented by making the initial portion of the left atrial incision adjacent to the right superior pulmonary vein in the previously dissected Sondergaard (Waterston) interatrial groove. Once again, this incision will later be extended during the division of the left atrium into cardiac and pulmonary cuffs.

This left atrial incision will provide excellent left heart decompression so long as a sucker is immediately placed 
through it into the left atrium. Once again, the primary advantage of this vent site location is the elimination of the need for suture closure of the left atrial appendage vent site.

- The inferior vena cava is then vented by hemitransection.

The inferior vena cava is vented last so that the more critical left atrial venting maneuver is not obscured by the large volume of blood that invariably fills the pericardium after venting of the inferior vena cava. In contrast to the left inferior pulmonary vein or midwall of the left atrium, which are both in the bottom of the pericardial well, the inferior vena cava is easily located - even with flooding of the field by the left atrial vent site- by running two fingers down the diaphragm at the inferolateral aspect of the pericardial well.

- The ligation of the superior vena cava, venting of the left atrium, and venting of the inferior vena cava combine to optimize the decompression of the left ventricle such that the aorta can now be crossclamped without fear of ventricular distention.

Left ventricular distention invariably occurs if the aortic crossclamp is applied (thus preventing left ventricular emptying) as the first step in the organ preservation procedure, before reduction in left ventricular return.

- Both cardiac and pulmonary preservation fluids are infused after application of the aortic crossclamp. The cardioplegic solution is infused at a pressure of approximately $80 \mathrm{~mm} \mathrm{Hg}$, whereas the pulmonary perfusate is administered into the pulmonary arteries through the high-flow perfusion cannula at gravity pressure only.

- A slight reduction in ventilatory tidal volume allows the instillation of a larger volume of ice saline slush into both pleural spaces, thereby optimizing topical cooling of the lungs. External cardiac cooling is facilitated by the pericardial instillation of cold saline solution.

- Two large bags (2.8 L each) of cold Perfadex lung preservation fluid (Vitrolife AB, Gothenburg, Germany) are infused until only $1 \mathrm{~L}$ remains in the final bag.

- A minimum of $1 \mathrm{~L}$ of cold crystalloid cardioplegic solution is administered to the heart. Inasmuch as $1 \mathrm{~L}$ of cardioplegic solution almost invariably finishes infusing before the pulmoplegia infusion, an extra 1-L bag of cardioplegic solution (two total) should be connected in line for continued infusion. This allows the continued distention of the ascending aorta by the sustained administration of cardioplegic solution for the duration of the pulmoplegia administration.

The persistent distention of the ascending aorta by the continued cardioplegic infusion prevents inadvertent coronary perfusion by the lung perfusate that is returning to the left side of the heart from the lungs.
- The adequacy of cardioplegic solution delivery must be assured by a rapid cessation of cardiac contractile and electrical activity. The adequacy of aortic root pressure and absence of ventricular distention must be continually monitored during infusion of cardioplegic solution.

Left ventricular distention during infusion of cardioplegic solution mandates immediate attention. It can be most efficiently eliminated by a short release of the aortic crossclamp with simultaneous gentle manual compression of the heart. Repeated distention of the heart suggests that either aortic insufficiency (primary or iatrogenic) is present or that the vena caval and left atrial venting maneuvers (see above) are not adequate and in need of immediate further attention.

- In a similar fashion, the free flow of gradually clearing Perfadex solution from the left atrial vent site must be monitored during pulmoplegia infusion. Equal distribution of perfusate to both lungs must be assured.

Both lungs should "blanch" equally with the administration of pulmoplegia. The midline positioning of the tip of the pulmonary artery perfusion cannula must be intermittently confirmed to assure that differential flow to one lung over the other is not occurring secondary to inadvertent cannula displacement.

\section{THORACIC ORGAN EXCISION}

- After the completion of thoracic organ preservation infusions, the apex of the heart is elevated and the inferior vena caval transection is completed.

- The left atrial incision is started halfway between the left inferior pulmonary vein and the atrioventricular groove (coronary sinus).

- Further extension of the initial left atrial incision is carried out parallel to the atrioventricular groove-toward the base of the left atrial appendage on the left and toward the inferior edge of the inferior vena cava on the right. A minimum of a 1-cm cuff (measured from the atrioventricular groove) must be retained on the left atrium for the subsequent cardiac implant procedure. The left atrial incision is then completed from inside the left atrium. Accordingly, all four pulmonary vein orifices can be visualized with preservation of optimal left atrial cuff lengths on both the heart and lung blocks.

- The superior vena cava and azygos vein are transected just below the previously tied supra-azygos superior vena caval silk ligature.

- The innominate and left carotid arteries are divided. The aortic arch is transected beyond the left carotid with care being taken to avoid injury to the pulmonary artery near the attachment of the ligamentum arteriosum.

- The main pulmonary artery is transected at the level of the pulmoplegia cannulation site. 
- The heart is removed from the field and submerged in a sterile plastic transportation canister that is filled with cold preservation solution. No ice is placed in the canister.

- The heart is examined to exclude previously undetected pathologic conditions or surgical damage.

- The plastic canister lid is secured and the canister is sealed in a sterile plastic bag that is filled with enough sterile ice slush to cover the canister.

- The bagged canister is then sealed in a final sterile plastic transport bag. The entire cardiac preservation and transport package is carefully labeled, placed in an ice chest, and covered with ice for transportation. The electrocardiogram, echocardiogram, donor lymph tissue (if applicable), and any other pertinent donor information are secured in or to the ice chest. The ice chest is closed and securely taped to prevent accidental opening during transport.

- The heart recipient implant team is then informed that the heart has been successfully procured. Any abnormalities found on the final postprocurement cardiac examination are discussed with the cardiac implant team and the estimated donor organ arrival time is updated.

- The lung preservation is completed after the cardiac excision. Uniform distribution of preservation fluid to the double lung block is ensured by an additional $250 \mathrm{~mL}$ retrograde flush of Perfadex solution into each of the four pulmonary vein orifices (retrograde flush total $=1 \mathrm{~L}$ ).

The retrograde flush is performed by infusing Perfadex solution through an inflated Foley catheter with its tip gently positioned in each individual pulmonary vein orifice. The return of clear retrograde flush solution into the ipsilateral pulmonary artery assures that the infusion is proceeding correctly. Occasionally, a pulmonary embolus is flushed from the pulmonary artery during this infusion.

- Excision of the double lung block then begins in the mediastinum at the level of the aortic arch vessels. All mediastinal tissue is divided down to the level of the mid-trachea.

- After donor extubation, the trachea is divided several centimeters above the carina between two TA-30 staple lines (Auto Suture Company Division, United States Surgical Corporation, Norwalk, Conn). No attempt is made to distend the lungs before tracheal stapling. The endotracheal tube is simply removed and the trachea is stapled and divided.

- The esophagus is mobilized and divided with a GIA stapler (Auto Suture) after nasogastric tube removal.

- All remaining mediastinal tissue is then divided straight back to the thoracic spine.

- The mediastinum/lung block is gently lifted up as it is sharply dissected directly off the spine down to the level of the mid-thorax.
- Attention is then turned to the lower thorax, where the pericardium is divided near its diaphragmatic attachments.

Care must be taken to avoid pulmonary parenchymal injury during this step inasmuch as the inferior pulmonary ligaments tether the basal segments of both lower lobes near the diaphragm, directly below the line of pericardial division.

- Both inferior pulmonary ligaments are carefully mobilized and divided.

Division of the inferior pulmonary ligaments is best performed by reaching behind the lung and gently retracting each lower lobe anteriorly. The tethering inferior pulmonary ligament can then be safely divided under direct visualization.

- The lower esophagus is encountered directly behind the pericardium. It is divided at this level with a second application of the GIA stapler.

- All remaining mediastinal tissue is divided straight back to the spine.

- The remaining mediastinal connections are then divided sharply off the spine and the double lung block is removed from the thoracic cavity.

- The double lung block is placed in a sterile plastic transport bag filled with cold Perfadex preservation solution (no ice). The bag is briefly supported in a basin on the back table as the lungs are examined for unexpected pathologic conditions, inadequate cuff margins, or surgical damage.

- If the two lungs are destined for two separate single lung transplant procedures at two different institutions, they are best removed from the donor en bloc and separated on the back table.

The separation of the two single lung blocks is initiated by sharp excision of the esophagus and aorta. The left atrial and pulmonary arterial cuffs are divided in the midline. The rest of the mediastinal tissue is divided in the midline, leaving the airway isolated. The airway is divided with as little disruption of the vascular blood supply to the trachea and bronchi as possible. The division is completed by dissecting the longer left main stem bronchus near the carina and dividing it between two TA-30 staple lines. Care is taken to stay clear of the carina, while at the same time preserving as much of the left main stem bronchus as possible.

- After the final examination of the lungs, the sterile plastic transport bag is sealed and placed into a second sterile bag. Ice slush is added to the second plastic transport bag to assure coverage of the first. The second bag is then sealed. 
- The double-bagged lung block is then secured inside a third sterile plastic transport bag.

- The lung transport bag assembly is carefully labeled, placed in a secure ice chest, and covered with ice. The donor chest x-ray film, lymph tissue (if applicable), and other pertinent information are secured in or to the ice chest. The ice chest is closed and securely taped for transport.

- The lung recipient implant team is then informed that the lungs have been successfully procured. Any abnormalities found on the final postprocurement lung examination are discussed with the implant team with an update on estimated donor organ arrival time.

\section{ISOLATED HEART PROCUREMENT}

When the lungs are not being procured, the cardiac procurement methodology can be simplified slightly from the combined heart and lung procurement procedure described above.

- During the initiation of the cardiac preservation, the left atrium is vented by transecting the left inferior pulmonary vein instead of the left atrial appendage.

- During the cardiac excision, the left atrium is detached posteriorly by simply transecting each of the pulmonary veins at the pericardium.

- Similarly, the pulmonary artery transection is completed by individually dividing the right and left pulmonary arteries just proximal to their exit from the pericardium.

Bilateral pulmonary artery transection is best performed as the final step of the cardiac extraction. The entire cardiac block can then be retracted in a caudad direction such that the heart is tethered only by both pulmonary arteries, which can then be safely transected adjacent to the pericardium.

\section{ISOLATED LUNG PROCUREMENT}

When cardiac procurement is not being performed, minor variations in the above-described combined heart and lung procurement procedure allow for further optimization of lung block pulmonary artery and left atrial cuffs.

- During the preparation for pulmonary preservation, the pulmoplegia cannula can be placed in the proximal main pulmonary artery near the pulmonary valve instead of distally near the pulmonary artery bifurcation. When placed more proximally, the tip of the pulmoplegia cannula is directed posteriorly toward the bifurcation of the main pulmonary artery.

- The heart is completely excised before lung extraction with one modification of the combined heart and lung procurement procedure described above. Inasmuch as there is no need to preserve a left atrial cuff for cardiac implantation, the left atrial incision is initiated immediately adja- cent to the interatrial groove posteriorly. This maneuver optimizes the size of the left atrial cuff that remains attached to the pulmonary veins. The incision is continued in the atrioventricular groove circumferentially around the heart, leaving the entire left atrium with the lung block.

\section{WHOLE HEART-LUNG BLOCK PROCUREMENT}

Procurement of the entire heart-lung block for transplantation into a single recipient obviously simplifies the combined heart and lung procurement technique described above.

- Only the supra-azygos superior vena caval and tracheal portions of the thoracic organ dissection need be performed.

- The thoracic organ preservation necessarily involves the infusion of both cardiac and pulmonary preservation solutions-excluding the retrograde pulmonary vein perfusion.

- Only three of the main steps of the previously described thoracic organ excision are required: the midtracheal stapling and transection of the airway, superior and inferior vena caval division, and resection of the entire heartlung block from its mediastinal attachments.

\section{CONCLUSION}

This review has been specifically aimed at the training and experience levels of the cardiothoracic surgical fellow and junior faculty. They represent the future of thoracic organ procurement, and their generation will be responsible for introducing and fostering the necessary changes in attitude regarding the sharing of procurement responsibilities. It is understood that the circumstances and demands of each donor and each thoracic transplant recipient are unique, thereby mandating some degree of flexibility in any proposed standardization of the thoracic organ procurement procedure. After further dialogue, it is anticipated that the presence of a thoracic organ procurement standard will allow thoracic organ transplant teams across the continent to become more comfortable with shared procurement responsibilities. Improved patient care, optimized organ usage, and enhanced efficiency in the application of available transplantation resources will necessarily result from any such efforts.

I acknowledge the excellent contributions to this editorial by the cardiothoracic fellows in the Division of Cardiothoracic Surgery at Washington University Medical School including Laura Adam, MD, Chad Denlinger, MD, Felix Fernandez, MD, Elbert Kuo, MD, Spencer Melby, MD, Varun Puri, MD, and Victor Van Berkel, MD. I would also like to acknowledge the excellent content suggestions and gracious editorial assistance of Christina C. Pasque, MD. 